\title{
Entrenamiento físico de alta intensidad en maratonistas produce mayor remodelado cardíaco y reduce respuesta de estrés oxidativo
}

\author{
Julián Vega ${ }^{1}$, Felipe Contreras-Briceño ${ }^{2}$, Rodrigo Saavedra ${ }^{1}$, Rodrigo Fernández ${ }^{1}$, Sebastián Herrera ${ }^{1}$, Manuel Salinas ${ }^{1}$, Paz Godoy $^{1}$, \\ María Paz Ocaranza ${ }^{1}$, Jorge Jalii ${ }^{1}$, Sergio Lavandero ${ }^{3}$, Mario Chiong ${ }^{3}$, Pablo Castro ${ }^{1}$, Roberto Urzúa ${ }^{4}$, Mario Muñoz ${ }^{4}$, Ricardo Zalaquett ${ }^{1}$ y \\ Luigi Gabrielli ${ }^{1}$.
}

\author{
1 Advanced Center for Chronic Diseases. Escuela de Medicina. Pontificia Universidad Católica de Chile. \\ 2 Kinesiología. Departamento Ciencias de la Salud. Facultad de Medicina. Pontificia Universidad Católica de Chile \\ 3 Advanced Center for Chronic Diseases. Facultad de Química y Farmacia y Medicina. Universidad de Chile. \\ 4 Hospital del Trabajador
}

Conflictos de interés: Los autores declaran que no tienen conflictos de intereses.

Introducción: El ejercicio físico reduce la mortalidad cardiovascular y genera remodelado cardíaco. Altas cargas de entrenamiento pueden generar remodelado cardíaco adverso. Biomarcadores (BMC) de inflamación Interleukina 6 (IL-6) y de estrés oxidativo Malondialdehído (MDA), potencialmente pueden caracterizar la respuesta al esfuerzo.

Objetivo: Evaluar actividad de IL-6 y MDA en respuesta a una maratón en atletas con distinto nivel de entrenamiento y remodelado cardíaco asociado.

Sujetos y Métodos: Estudio prospectivo, simple ciego, incluyó 16 atletas que completaron la maratón de Santiago (42 k), separados según entrenamiento previo, grupo 1 (G1, n: 8): Alto $\geq 100 \mathrm{~km} / \mathrm{semana} \mathrm{y}$ grupo 2 (G2, n: 8): Bajo $<100$ km/semana). Se obtuvo pre y post maratón: niveles de IL-6, MDA y ecocardiografía Doppler transtorácica (ETT); cuantificando cámaras cardíacas izquierdas, derechas y deformación del ventrículo izquierdo (strain longitudinal). Se utilizaron las pruebas de Mann-Whitney, Wilcoxon y Krus-
kal-Wallis.

Resultados: Edad G1: $38.13 \pm 7.18$ años vs G2: $40.38 \pm 6.63$ años (NS). Tiempo maratón G1: $185.75 \pm 14.87 \mathrm{~min}$ vs $\mathrm{G} 2: 219.75 \pm 24.92 \min (\mathrm{p}<0.01)$. Masa del ventrículo izquierdo G1: $91 \pm 21 \mathrm{~g} / \mathrm{m} 2$ vs G2: $73 \pm 12 \mathrm{~g} / \mathrm{m} 2 \quad(\mathrm{p}<0.01)$. Volumen aurícula izquierda G1: $39.4 \pm 12.6 \mathrm{ml} / \mathrm{m} 2$ vs $30.6 \pm 4.6 \mathrm{ml} / \mathrm{m} 2(\mathrm{p}<0.01)$. FEVI G1: $55.8 \pm 3.3 \%$ vs G2: $58.6 \pm 6.7 \%$ (NS). MDA G1: PRE $0.17 \pm 0.13 \mathrm{uM} / \mathrm{L}$, POST $0.67 \pm 0.59 \mathrm{uM} / \mathrm{L}$, G2: PRE $0.29 \pm 0.24 \mathrm{uM} / \mathrm{L}$, POST $1.01 \pm 1.15 \mathrm{uM} / \mathrm{L}$ $(\mathrm{p}<0.01)$. IL-6 G1: PRE $2.50 \pm 1.35 \mathrm{pg} / \mathrm{ml}$, POST 93.91 $\pm 27.23 \mathrm{pg} / \mathrm{ml}$ vs G2: PRE $4.65 \pm 5.89 \mathrm{pg} / \mathrm{ml}$, POST $97.83 \pm 30.72 \mathrm{pg} / \mathrm{ml}$ (NS).

Conclusión: El ejercicio físico aumenta los BMC de inflamación y estés oxidativo (IL-6, MDA). Un entrenamiento físico de alta intensidad disminuye la respuesta de estrés oxidativo y se asocia a un mayor remodelado cardíaco.

Palabras clave: estrés oxidativo; remodelamiento ventricular; deporte; maratón. 


\section{High-intensity training in marathon runners increases cardiac remodeling and diminishes oxidative stress response}

Background: Exercise reduces cardiovascular mortality and generates cardiac remodeling. High training loads can induce adverse cardiac remodeling, and its associated cardiac remodeling. Therefore, interleukin 6 (IL 6) and malondialdehyde (MDA), biomarkers of inflammatory response and oxidative stress respectively, may have a role in stratifying this risk.

Objective: To assess the activity of IL-6 and MDA in response to a marathon race in athletes with different previous training status

Subjects And Methods: Prospective, single-blind study involving 16 male athletes that finished the Santiago Marathon ( $42 \mathrm{k}$ ), allocated into two groups according to their previous training: Group 1 (G1, n: 8) with high training ( $\geq 100 \mathrm{~km} /$ weekly) and Group 2 (G2, n: 8) with low training $(<100 \mathrm{~km} /$ weekly). Before and after the race serum levels of IL-6, MDA and transthoracic Doppler echocardiography for cardiac chamber quantification and left ventricle deformation (longitudinal strain) were measured.
Mann-Whitney, Wilcoxon, and Kruskal-Wallis tests were used to assess statistical significance.

Results: Age G1: $38.13 \pm 7.18$ years-old vs G2: $40.38 \pm 6.63$ years-old (NS). Marathon finishing time G1: $185.75 \pm 14.87 \mathrm{~min}$ vs G2: $219.75 \pm 24.92$ $\min (\mathrm{p}<0.01)$. Left ventricle mass $\mathrm{G} 1: 91 \pm 21 \mathrm{~g} / \mathrm{m} 2$ vs $\mathrm{G} 2: 73 \pm 12 \mathrm{~g} / \mathrm{m} 2(\mathrm{p}<0.01)$. Left atrium volume G1: $39.4 \pm 12.6 \mathrm{ml} / \mathrm{m} 2$ vs $30.6 \pm 4.6 \mathrm{ml} / \mathrm{m} 2(\mathrm{p}<0.01)$. LVEF G1: $55.8 \pm 3.3 \%$ vs G2: $58.6 \pm 6.7 \%$ (NS). MDA G1: PRE $0.17 \pm 0.13 \mathrm{uM} / \mathrm{L}$, POST $0.67 \pm 0.59 \mathrm{uM} / \mathrm{L}$, G2: PRE $0.29 \pm 0.24 \mathrm{uM} / \mathrm{L}$, POST $1.01 \pm 1.15 \mathrm{uM} / \mathrm{L}$ $(\mathrm{p}<0.01)$. IL-6 G1: PRE $2.50 \pm 1.35 \mathrm{pg} / \mathrm{ml}$, POST $93.91 \pm 27.23$ vs G2: PRE $4.65 \pm 5.89 \mathrm{pg} / \mathrm{ml}$, POST $97.83 \pm 30.72 \mathrm{pg} / \mathrm{ml}$ (NS).

Conclusion: Physical exercise generates a rise in biomarkers (IL-6, MDA). Athletes with high-intensity training level have a diminished oxidative stress response post effort and greater cardiac remodeling. Keywords: oxidative stress, ventricular remodeling, sports, running. 


\section{Introducción:}

El ejercicio físico tiene un reconocido rol en la prevención de las enfermedades cardiovasculares ${ }^{1}$, evidenciando una relación inversa dosis dependiente entre actividad física y mortalidad ${ }^{2,3}$. Dicha reducción en mortalidad es producto de varios mecanismos, entre los cuales destaca un remodelado cardíaco favorable $e^{4,5}$.

Este remodelado se genera por medio de una expresión genómica que gatilla efectos moleculares, intersticiales y celulares, que a su vez producen modificaciones estructurales, eléctricas y funcionales en el sistema cardiovascular $^{6}$. Estas modificaciones son objetivables tempranamente, a meses de iniciado el entrenamiento, y son benignas y reversibles ${ }^{5}$. Ellas incluyen aumento del diámetro de ambos ventrículos 7 , aumento del grosor parietal del ventrículo izquierdo ${ }^{8}$, incluso, en ocasiones asociado a mayor trabeculación de éste ${ }^{9}$ y dilatación auricular izquierda ${ }^{4}$ con función diastólica normal.

En el extremo opuesto, existen atletas que al ser sometidos a altas cargas de entrenamiento, experimentan un remodelado cardiaco que sobrepasa la barrera de lo fisiológico y que ha sido asociado a efectos deletéreos, condición denominada miocardiopatía de "Pheidippides"10 y que incluye: hipertrofia ventricular izquierda asociada a fibrosis miocárdica ${ }^{11}$, aumento en la incidencia de fibrilación auricular ${ }^{12}$, aumento de la ateromatosis coronaria ${ }^{13}$ y mayor remodelado ventricular derecho. Este remodelado adverso es un elemento de aparición relativamente tardía, siendo necesario contar con predictores más precoces de respuesta frente al ejercicio. En este contexto, se ha estudiado la dinámica de diversos biomarcadores frente al ejercicio y que podrían ser predictores tempranos de mala adaptación cardíaca al entrenamiento. Algunos de estos marcadores estudiados carecen de significado patológico, como son el alza transitoria post ejercicio de las troponinas ${ }^{14}$ y del péptido natriurético (pro-BPN) ${ }^{15}$, que se pueden presentar hasta en un tercio de los maratonistas ${ }^{10}$. En tanto que otros marcadores relacionados a actividad inflamatoria (Interleukina 6) y estrés oxidativo (Malondialdehído) ${ }^{16}$ pueden tener un potencial significado patológico y se comentan a continuación.

\section{Interleukina-6 (Il-6)}

La IL-6 es una citokina mediadora de la respuesta de fase aguda que, además, es sintetizada por el músculo esquelético contráctil, siendo liberada al intersticio y a la circulación sistémica en respuesta al ejercicio ${ }^{17,18}$. Su concentración plasmática queda determinada por la intensidad y duración del ejercicio, independiente del tipo de ejercicio realizado, objetivando un aumento en sus niveles de hasta 100 veces posterior a una maratón ${ }^{19}$. En cuanto a su cinética, la IL-6 alcanza un máximo dentro las primeras horas de iniciado el ejercicio, disminuyendo rápidamente al finalizar la competencia. Su rol es modular la respuesta inmunológica y metabólica del organismo frente al esfuerzo, como, por ejemplo, aumentando la entrega de glucosa por parte del hígado y estimulando la lipólisis. Ha sido propuesto que el entrenamiento físico sostenido produce una disminución en los niveles basales de IL-6, además de frenar su alza post ejercicio ${ }^{20}$, siendo este fenómeno uno de los mecanismo bioquímicos que se ha sugerido como adaptación en atletas entrenados ${ }^{17}$. Además, se ha propuesto que un alza desmedida, tanto de forma basal, como post actividad física, puede identificar a sujetos con respuestas "mal adaptativas" frente al ejercicio.

\section{Malondialdehído (MDA)}

La peroxidación lipídica es el proceso de degradación oxidativa de los lípidos, en el cual los radicales libres degradan ácidos grasos de la membrana celular. El estrés oxidativo refleja un desbalance entre la producción de especies de oxígeno reactivo y la actividad y cantidad de las defensas antioxidantes del organismo, que cuando son sobrepasadas, se produce daño tisular y celular. El MDA es un producto final de este proceso y puede ser medido en plasma, tejidos y orina mediante diversas técnicas. Es el marcador que con más frecuencia se utiliza para evaluar el nivel de estrés oxidativo en atletas y al igual que la IL-6 tiene una cinética de liberación aguda en relación a la actividad física.

La relación entre ejercicio y estrés oxidativo es compleja, ya que varía dependiendo del tipo de ejercicio, su duración, la intensidad, el nivel de entrenamiento previo y de la capacidad antioxidante total de cada atleta ${ }^{21}$, reportándose que un mayor entrenamiento físico aumenta la capacidad antioxidante total 22 .

\section{Biomarcadores, Remodelado Cardíaco y Ejercicio}

La cinética de estos biomarcadores en relación al ejercicio ha sido estudiada, pero no existe claridad en cómo la intensidad de entrenamiento previo influye en la magnitud de su alza, ni de la asociación con el grado de remodelado cardiaco. Aspecto de interés, pues actualmente un creciente número de atletas realiza actividad física de alta intensidad, existiendo en este grupo individuos biológicamente susceptibles que presentarán un remodelado cardiaco patológico asociado a eventos clínicos adversos. Y es aquí donde los biomarcadores descritos pueden te- 
ner un rol en la identificación y estratificación de dichos deportistas.

El objetivo de este trabajo es evaluar la actividad de IL-6 y MDA en respuesta a una maratón en atletas con distinto nivel de entrenamiento previo y el remodelado cardíaco asociado.

\section{Sujetos y Métodos:}

\section{Sujetos}

La muestra incluyó 16 atletas hombres entre 18 y 50 años, que completaron la maratón de Santiago de Chile del día 2 de abril de 2017 con un recorrido total de $42 \mathrm{~km}$. Todos habían terminado al menos tres maratones dentro de los últimos cinco años.

Los criterios de exclusión fueron: HTA (presión arterial en reposo $>140 / 90 \mathrm{mmHg}$ en 2 instancias separadas, dislipidemia (colesterol total $>200 \mathrm{mg} / \mathrm{dl}$, LDL $>100 \mathrm{mg} / \mathrm{dl}$, HDL $<40 \mathrm{mg} / \mathrm{dl}$, triglicéridos $>150 \mathrm{mg} / \mathrm{dl}$ ), diabetes mellitus, resistencia a la insulina (HOMA $>2.5$ ), tabaquismo en cualquier grado), enfermedad cerebrovascular, abuso de alcohol o drogas, uso de suplementos nutricionales, deterioro de la función renal definido como una tasa de filtración glomerular $<60 \mathrm{ml} / \mathrm{min}$, historia familiar de muerte súbita, falla hepática, enfermedad autoinmune, neoplasia activa, enfermedad pulmonar obstructiva cró-

Figura 1. Diagrama del estudio (ETT: Ecocardiografía transtorácica, BMC: Biomarcadores (IL-6, MDA)

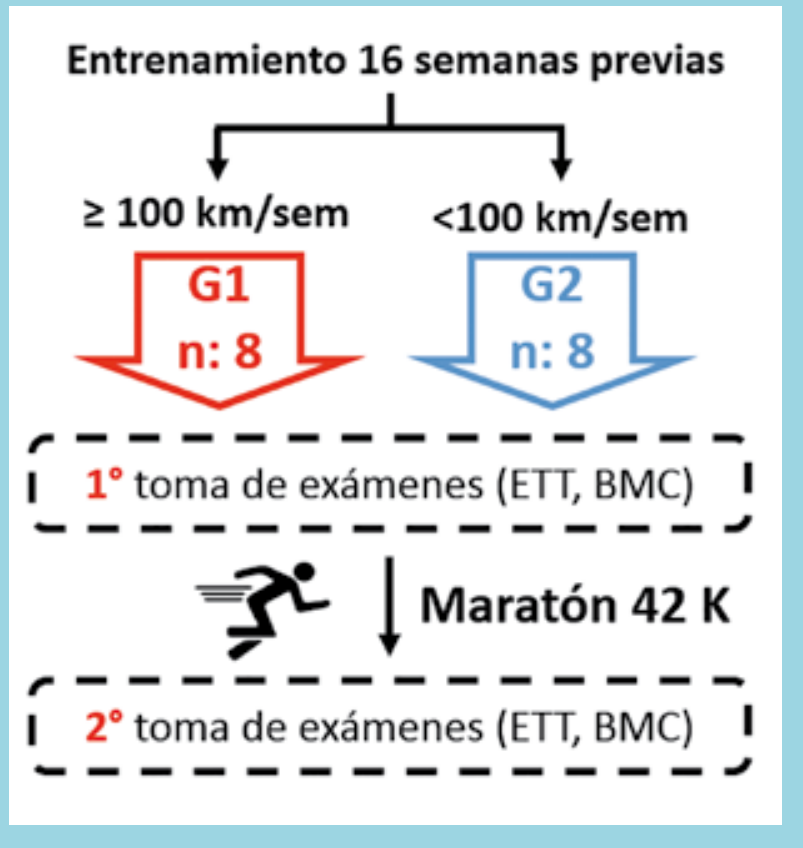

nica, enfermedades que alteren niveles de los biomarcadores estudiados (inflamación aguda o enfermedad infecciosa en las 4 semanas previas al estudio) y el uso de los siguientes medicamentos: cualquier anti-hipertensivo, anorexígenos, anti-depresivos, antibióticos.

El estudio contó con la aprobación del comité de ética de las instituciones participantes y se encontraba en concordancia con la declaración de Helsinki.

\section{Métodos:}

Estudio prospectivo, simple ciego. A todos los atletas se les realizó de manera basal (durante la semana previa al evento) e inmediatamente al finalizar la maratón (dentro los 5 min de finalizar la prueba), una punción venosa para

\begin{tabular}{|c|c|}
\hline Variable & Unidad \\
\hline \multicolumn{2}{|l|}{ BIODEMOGRÁFICAS Y CLÍNICAS } \\
\hline Edad & años \\
\hline Peso & $\mathrm{kg}$ \\
\hline Estatura & $\mathrm{cm}$ \\
\hline \multicolumn{2}{|l|}{ VARIABLES DE ENTRENAMIENTO } \\
\hline Años de entrenamiento & Años \\
\hline Tiempo de maratón & Minutos \\
\hline \multicolumn{2}{|l|}{ ECOCARDIOGRÁFICAS } \\
\hline Ventrículo izquierdo & $\mathrm{mm}$ \\
\hline Grosor del septum interventricular & $\mathrm{mm}$ \\
\hline Grosor de la pared posterior & $\mathrm{mm}$ \\
\hline Diámetro de fin de diástole & $\mathrm{mm}$ \\
\hline Diámetro de fin de sístole & $\mathrm{mm}$ \\
\hline Volumen de fin de diástole & $\mathrm{ml}$ \\
\hline Volumen de fin de sístole & $\mathrm{ml}$ \\
\hline Masa ventricular izquierda indexada & $\mathrm{g} / \mathrm{m} 2$ \\
\hline Fracción de eyección por Simpson biplano & $\%$ \\
\hline Strain longitudinal global (S.L.G.) bidimensional & $\%$ \\
\hline TAPSE del ventrículo derecho & $\mathrm{mm}$ \\
\hline Cambio de área fraccional del ventrículo derecho (FAC) & $\%$ \\
\hline \multicolumn{2}{|l|}{ Aurícula izquierda } \\
\hline Diámetro anteroposterior & $\mathrm{mm}$ \\
\hline Área en apical $2 \mathrm{C}$ y $4 \mathrm{C}$ & $\mathrm{cm} 2$ \\
\hline Volumen biplano indexado por método de discos (VIAI) & $\mathrm{ml} / \mathrm{m} 2$ \\
\hline \multicolumn{2}{|l|}{ Válvula mitral } \\
\hline Velocidad máxima de la onda $\mathrm{E}$ & $\mathrm{cm} / \mathrm{s}$ \\
\hline tiempo de desaceleración de la onda $\mathrm{E}$ & $\mathrm{cm} / \mathrm{s}$ \\
\hline Velocidad máxima de la onda $\mathrm{A}$ & $\mathrm{ms}$ \\
\hline \multicolumn{2}{|l|}{ BIOMARCADORES } \\
\hline IL-6 & $\mathrm{pg} / \mathrm{ml}$ \\
\hline MDA & UM/L \\
\hline
\end{tabular}




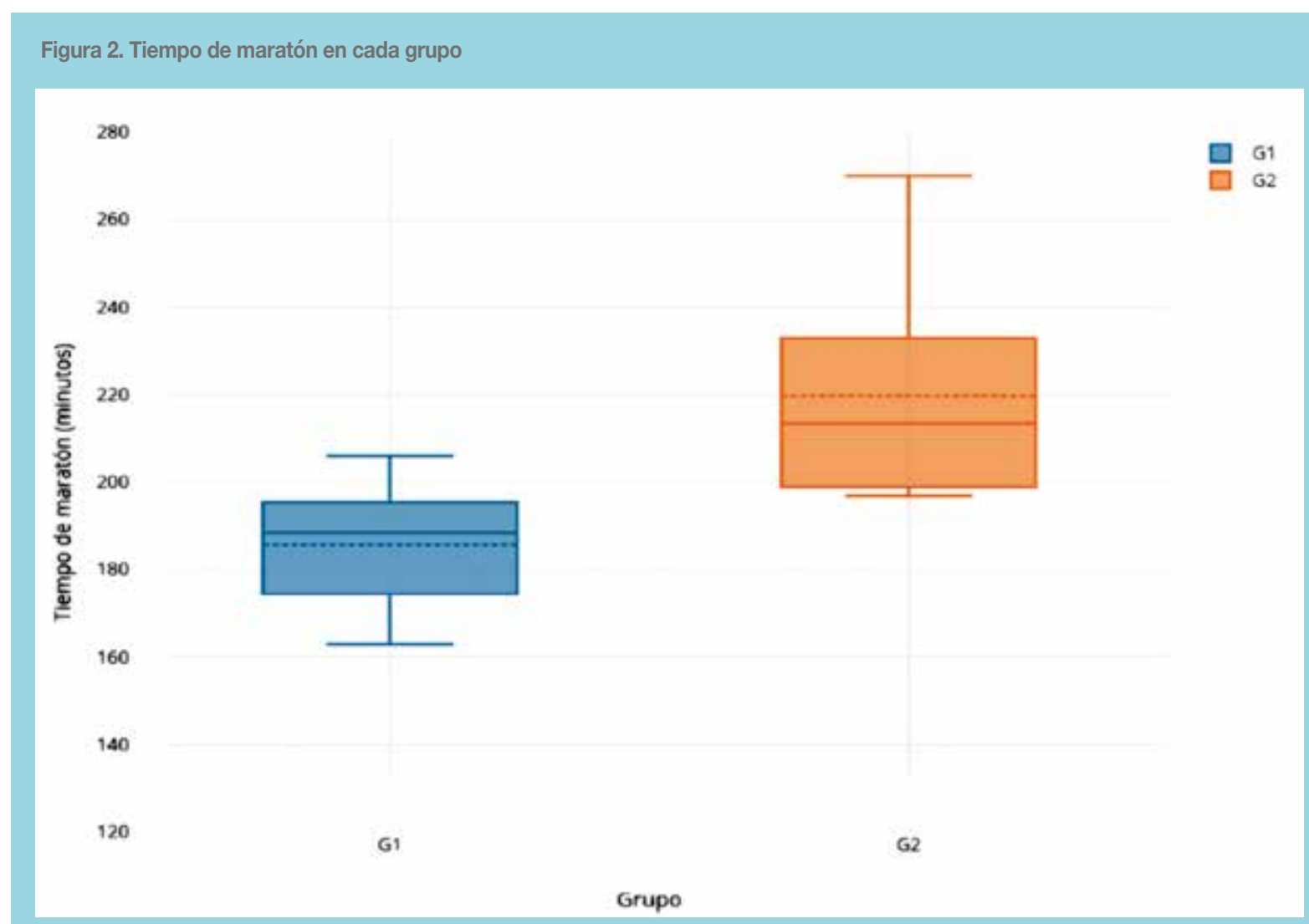

G1: Grupo 1: Alta carga de entrenamiento. G2: Grupo 2: Baja carga de entrenamiento

la toma de muestras séricas destinadas al análisis de los biomarcadores IL-6 y MDA. Asimismo, se realizó una ETT basal e inmediatamente al finalizar la competencia (en la línea de llegada). Mediante un cuestionario ad-hoc se estimó la intensidad auto-reportada de entrenamiento en las 16 semanas previas a la maratón, estableciendo así dos grupos de análisis; Grupo 1: alta carga de entrenamiento $\geq 100 \mathrm{~km} / \mathrm{semana}$ y Grupo 2 : baja carga de entrenamiento $<100 \mathrm{~km} / \mathrm{semana}$. El límite de $100 \mathrm{~km}$ para definir la carga previa de entrenamiento se fundamentó en protocolos institucionales sobre el entrenamiento en maratonistas (Figura 1).

\section{Ecocardiografía}

Se realizó un ETT pre y post maratón utilizando un equipo portátil Vivid I, General Electric, Healthcare, Horton, Norway, con un transductor de 1.57/3.54 MHz. Se adquirieron vistas tradicionales desde la ventana paraesternal, apical y subcostal, para la cuantificación de las cámaras cardíacas según las recomendaciones de la Asociación Americana de Ecocardiografía (ASE). Las variables re- gistradas se resumen en la Tabla 1. Utilizando el software del fabricante (EchoPAC BT 12 GE Healthcare, Horton, Norway) las imágenes fueron almacenadas para su análisis por ecocardiografistas expertos, ciegos al nivel de entrenamiento basal y a los resultados de los biomarcadores en los atletas.

\section{Biomarcadores}

Se determinaron, de forma basal e inmediatamente posterior a finalizar la maratón, niveles plasmáticos de malondialdehído (MDA) mediante la evaluación del contenido de substancias reactivas al ácido tiobarbitúrico (30) y niveles de IL-6 mediante técnica de ELISA con un kit comercialmente disponible. Las muestras fueron analizadas en el Laboratorio de Biología Molecular de la Pontificia Universidad Católica de Chile.

\section{Análisis estadístico}

Cada variable fue estudiada para evaluar su distribución normal. Las variables continuas se expresaron como medias \pm desviación estándar y las categóricas como cuentas 


\begin{tabular}{l||c|c|c|}
\hline \multicolumn{3}{|c|}{ Tabla 2. Variables biodemográficas y atléticas en cada grupo } \\
\hline Variable & Grupo 1 & Grupo 2 & $\mathrm{p}$ \\
\hline & $\mathrm{n}: 8$ & $\mathrm{n}: 8$ & \\
\hline Edad (años) & $38.13 \pm 7.18$ & $40.38 \pm 6.63$ & $\mathrm{NS}$ \\
\hline Peso (kg) & $66.38 \pm 9.26$ & $69.38 \pm 4.41$ & $\mathrm{NS}$ \\
\hline Estatura (cm) & $1.71 \pm 0.08$ & $1.73 \pm 0.05$ & $\mathrm{NS}$ \\
\hline Años de & $8.25 \pm 4.40$ & $7.25 \pm 5.60$ & $\mathrm{NS}$ \\
\hline entrenamiento & & & \\
\hline Tiempo de & $185.75 \pm 14.87$ & $219.75 \pm 24.92$ & $*$ \\
\hline maratón (min) & & & \\
\hline
\end{tabular}

NS: no significativo, $* * *$ : $<<0.01$

y porcentaje. Se analizaron de forma basal y post-ejercicio para cada grupo (alta carga de entrenamiento vs baja carga) las variables biodemográficas, ecocardiográficas y de los BMC que se resumen en la Tabla 1. Para establecer diferencias en las variables mencionadas se utilizaron las pruebas de Mann-Whitney, test de Wilcoxon y test de
Kruskal-Wallis, según correspondía. Se consideró significativo una $\mathrm{p}<0.05$.

\section{Resultados:}

La muestra quedó compuesta por 16 maratonistas, 8 en cada grupo según el nivel de entrenamiento previo (alto y bajo). La edad promedio fue de $39 \pm 6$ años y el promedio de los años de entrenamiento fue 7,6 \pm 4 años, no hubo diferencias biodemográficas significativas entre los grupos (Tabla 2). Todos los competidores finalizaron la maratón dentro de los tiempos establecidos por la organización. No obstante, el grupo altamente entrenado completo la maratón en un menor tiempo que el grupo con menor entrenamiento previo (185.75 \pm 14.87 vs 219.75 \pm 24.92 min, $\mathrm{p}<0.01$ ) (Figura 2). Los resultados de las variables ecocardiográficas y de los biomarcadores (IL-6, MDA) de cada grupo se resumen en la Tabla 3.

\section{Estrés Oxidativo (MDA)}

El grupo mejor entrenado (Grupo 1) no presentó un alza significativa de los niveles de MDA luego de la maratón: PRE- $0.17 \pm 0.13 \mathrm{uM} / \mathrm{L}$, POST $0.67 \pm 0.59 \mathrm{uM} / \mathrm{L}$, en cam-

Tabla 3. Variables ecocardiográficas y biomarcadores en cada grupo.

\begin{tabular}{|c|c|c|c|c|c|}
\hline \multirow[b]{2}{*}{ Variable } & \multicolumn{2}{|c|}{ Grupo 1 (n: 8) } & \multicolumn{2}{|c|}{ Grupo 2 (n: 8) } & \multirow[b]{2}{*}{$\mathrm{p}$} \\
\hline & PRE & POST & PRE & POST & \\
\hline \multicolumn{6}{|l|}{ ECOCARDIOGRÁFICAS } \\
\hline DTD (mm) & $48.1 \pm 4.22$ & $47.1 \pm 4$ & $46.2 \pm 3.5$ & $45 \pm 3.2$ & NS \\
\hline DTS (mm) & $29.5 \pm 4.2$ & $28.3 \pm 3.8$ & $28.3 \pm 3.3$ & $26.1 \pm 3.6$ & NS \\
\hline SIV (mm) & $9.1 \pm 1.1$ & $8.8 \pm 1.2$ & $8.5 \pm 1.2$ & $8.5 \pm 1$ & NS \\
\hline $\mathrm{PP}(\mathrm{mm})$ & $8.5 \pm 1.1$ & $8.8 \pm 1.1$ & $8.5 \pm 1.5$ & $8.2 \pm 0.8$ & NS \\
\hline FEVI\% & $55.8 \pm 3.3$ & $57.6 \pm 5.5$ & $58.6 \pm 6.7$ & $65 \pm 4.8$ & NS \\
\hline S.L.G. & $-20.6 \pm 1.1 .8$ & $-20.4 \pm 1.7$ & $-20.5 \pm 1.9$ & $-21.4 \pm 2$ & NS \\
\hline Masa VI (g/m2) & $91 \pm 21$ & - & $73 \pm 12$ & - & ** \\
\hline $\mathrm{VIAI}(\mathrm{ml} / \mathrm{m} 2)$ & $39.4 \pm 12.6$ & $39.5 \pm 12.2$ & $30.6 \pm 4.6$ & $31.1 \pm 4.7$ & ** \\
\hline Velocidad onda $E(\mathrm{~cm} / \mathrm{s})$ & $81.7 \pm 9.8$ & $84.4 \pm 12.6$ & $88 \pm 14.2$ & $68.9 \pm 12.8$ & NS \\
\hline Velocidad onda $A(\mathrm{~cm} / \mathrm{s})$ & $51.5 \pm 14.3$ & $73.9 \pm 12.9$ & $53.4 \pm 12.5$ & $75.26 \pm 18.6$ & NS \\
\hline TAPSE (mm) & $25.88 \pm 3.27$ & $25.25 \pm 3.01$ & $26.50 \pm 2.98$ & $24.25 \pm 3.45$ & NS \\
\hline FAC (\%) & $57.25 \pm 4.71$ & $53.88 \pm 10.99$ & $57.38 \pm 2.92$ & $50.13 \pm 8.77$ & NS \\
\hline \multicolumn{6}{|l|}{ BIOMARCADORES } \\
\hline MDA (uM/L) & $0.17 \pm 0.13$ & $0.67 \pm 0.59$ & $0.29 \pm 0.24$ & $1.01 \pm 1.15$ & ** \\
\hline IL-6 (pg/mL) & $2.50 \pm 1.35$ & $93.91 \pm 27.23$ & $4.65 \pm 5.89$ & $97.83 \pm 30.72$ & NS \\
\hline
\end{tabular}

S.L.G.: Strain longitudinal global del ventrículo izquierdo. VIAI: Volumen indexado auricular izquierdo. NS: no significativo, ***: $p<0.01$ 


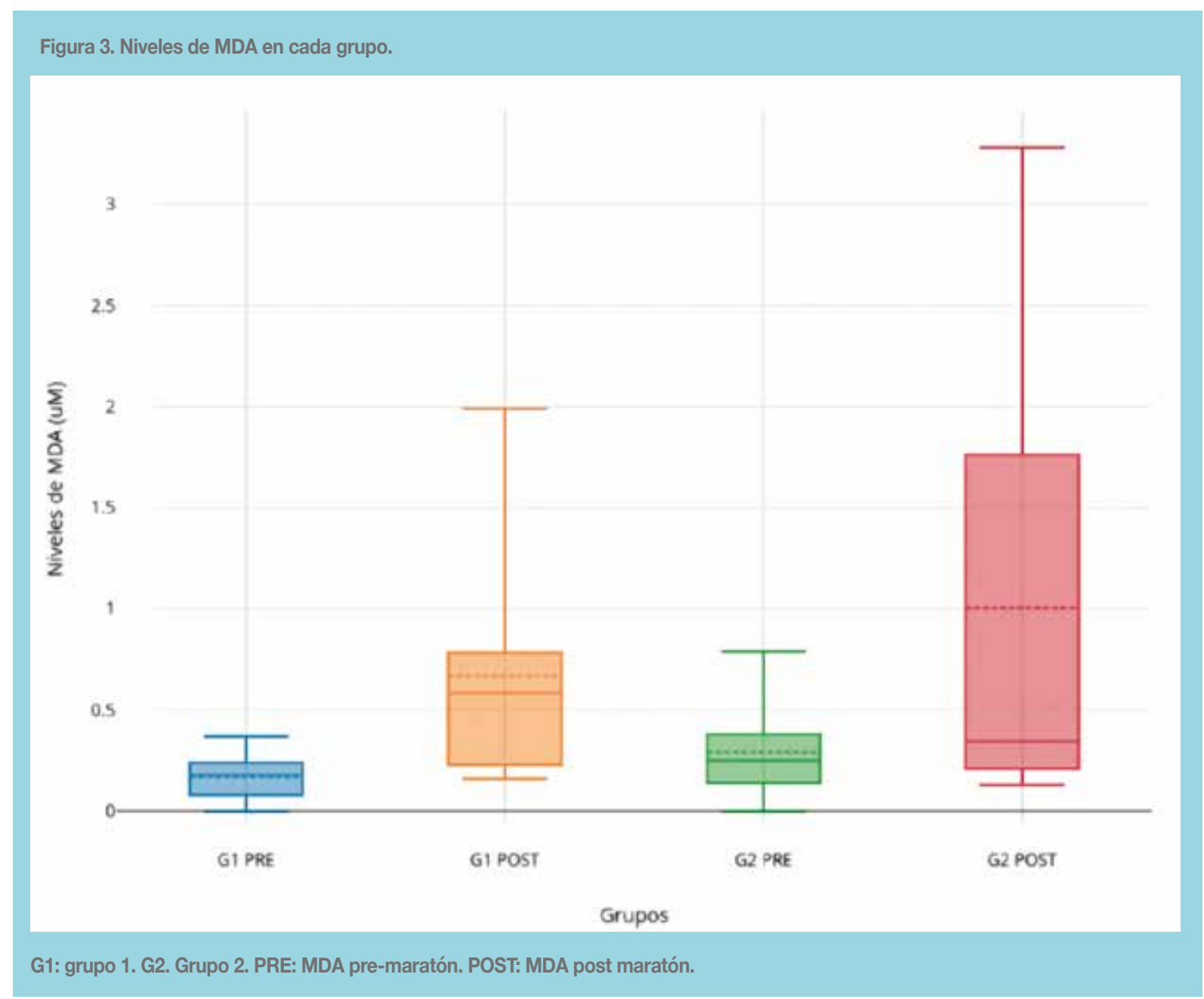

bio el grupo peor entrenado (Grupo 2) tuvo una significativa alza post maratón: MDA PRE-0.29 $\pm 0.24 \mathrm{uM} / \mathrm{L}$, POST $1.01 \pm 1.15 \mathrm{uM} / \mathrm{L}$ (Figura 3 ).

\section{Inflamación (Il-6)}

Basalmente los sujetos mejor entrenados presentaron una tendencia a tener menores niveles de IL-6 (Grupo 1: 2.50 $\pm 1.35 \mathrm{pg} / \mathrm{ml}$ vs Grupo 2: $4.65 \pm 5.89 \mathrm{pg} / \mathrm{ml}$ ), diferencia que alcanzó a ser estadísticamente significativa, así como tampoco se evidenciaron diferencias significativas en la magnitud del alza de IL-6 entre los grupos (Figura 4).

\section{Remodelado cardíaco}

El Grupo 1 presentó un mayor remodelado cardíaco evidenciado por una mayor masa ventricular izquierda $\mathrm{G} 1$ : $91 \pm 21 \mathrm{~g} / \mathrm{m} 2$ vs G2: $73 \pm 12 \mathrm{~g} / \mathrm{m} 2, \mathrm{p}<0.05$ (Figura 5) y un mayor volumen indexado auricular izquierdo:G1: $39.4 \pm$ $12.6 \mathrm{ml} / \mathrm{m} 2$ vs G2: $30.6 \pm 4.6 \mathrm{ml} / \mathrm{m} 2$, p $<0.05$ (Figura $6)$, sin asociarse a cambios en los parámetros funcionales del ventrículo izquierdo (FEVI, SLG) ni del ventrículo derecho (TAPSE, FAC) (Tabla 3). Sin embargo, no encontramos correlación entre el alza de los biomarcadores y el grado de remodelado cardíaco (MVI, VIAI).

\section{Discusión:}

Cada vez hay más pruebas de que el ejercicio intenso y prolongado puede inducir fibrosis miocárdica, fibrilación auricular, enfermedad aterosclerótica coronaria, mayor remodelado bi-ventricular y elevación de biomarcadores inflamatorios y otros asociados a daño miocárdico como son Interleukinas 6, 8 y 10, proteínas inflamatorias de macrófagos (MIP) 1, receptores de necrosis tumoral (TNF) y Rho kinasa entre otros ${ }^{19}$. Los resultados de esta serie muestran que después de un esfuerzo intenso como es correr una maratón, los niveles plasmáticos del marcador de estrés oxidativo MDA aumentan significativamente en los atletas peor entrenados. Dillard et $\mathrm{al}^{23}$ hacia fines de los años 1970 describió que el ejercicio físico produce la 


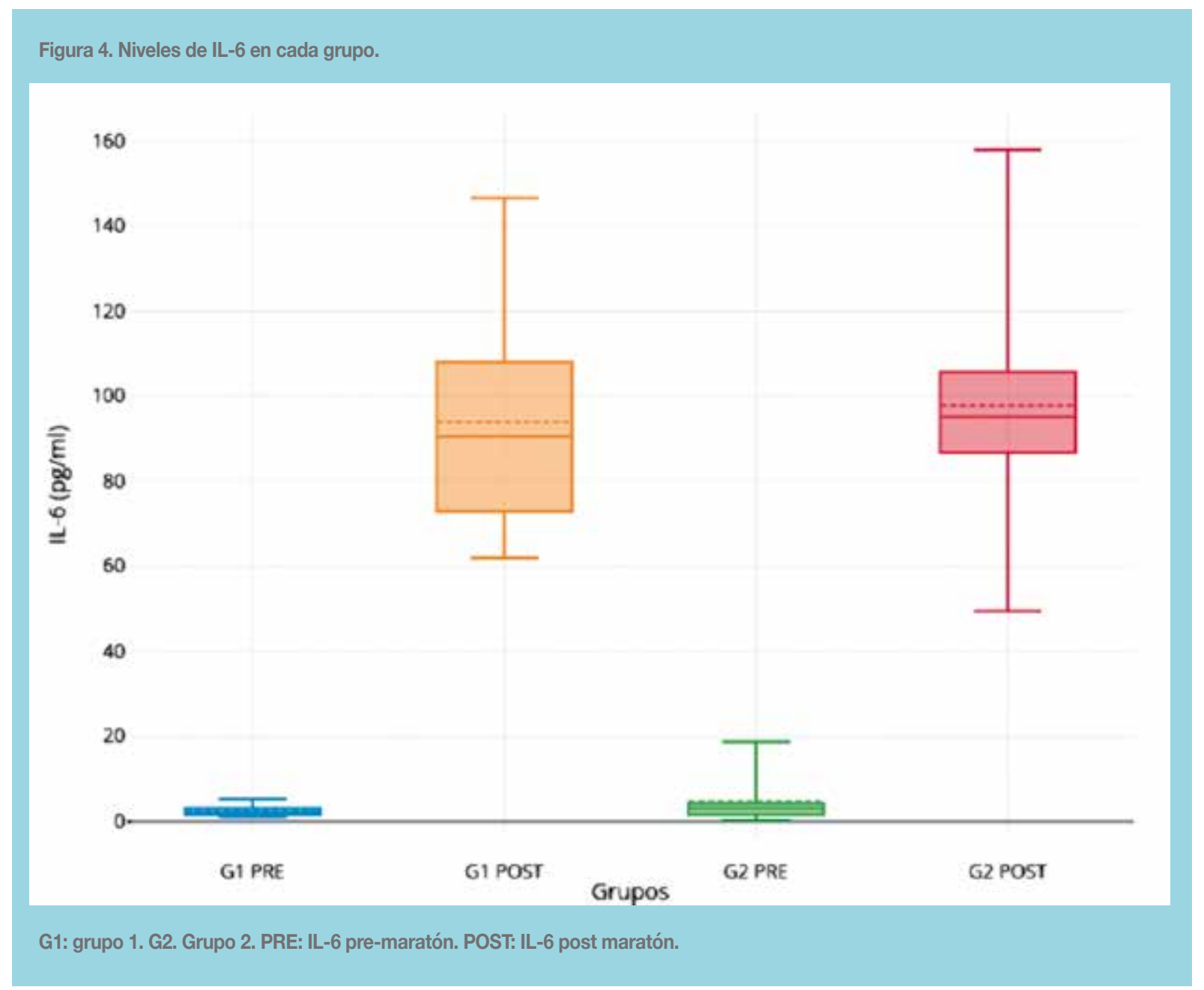

elevación de marcadores de estrés oxidativo en humanos. Desde entonces, se han publicado muchos trabajos que muestran la asociación entre diferentes marcadores de estrés oxidativo y diferentes tipos y dosis de ejercicio, sin una asociación clara con el remodelado cardiovascular o el impacto clínico ${ }^{24}$. Agregado a lo anterior, nuestros hallazgos sugieren que el nivel de entrenamiento previo influencia la respuesta de estrés oxidativo post-ejercicio. Específicamente, planteamos que en sujetos altamente entrenados existe una atenuación de dicha respuesta, la que podría ser explicada porque un mayor entrenamiento, asociado a un mejor remodelado cardíaco, produce una mayor actividad antioxidante total mediante el aumento de los niveles de superóxido dismutasa 25 , aspecto que actualmente se está evaluando en esta serie.

En este grupo de atletas determinamos, además, que el ejercicio intenso, también induce un aumento importante de los niveles plasmáticos de IL-6. Sin embargo, al igual que lo reportado por Scott et al $^{26}$, en este trabajo el nivel de entrenamiento previo no afectó la respuesta de IL-6 como otros autores han sugerido ${ }^{17}$. Como explicación a esto, se puede mencionar que la respuesta inflamatoria al ejercicio es compleja y, probablemente dependa, además del estado del entrenamiento, de otros factores como: la edad, el sexo y la disciplina del ejercicio practicada ${ }^{27}$. Los datos aquí publicados, permiten plantear futuras líneas de investigación relacionadas con el rol de algunos biomarcadores en identificar procesos de adaptación patológica y remodelado cardíaco adverso frente al ejercicio.

La cuantificación de esta respuesta oxidativa podría tener utilidad en identificar procesos de mala adaptación al ejercicio en corredores de maratón. Finalmente, es interesante evaluar si la suplementación con antioxidantes en el grupo peor entrenado es capaz de mitigar el alza de MDA evidenciada. 


\section{Figura 5. Remodelado cardíaco en cada grupo.}

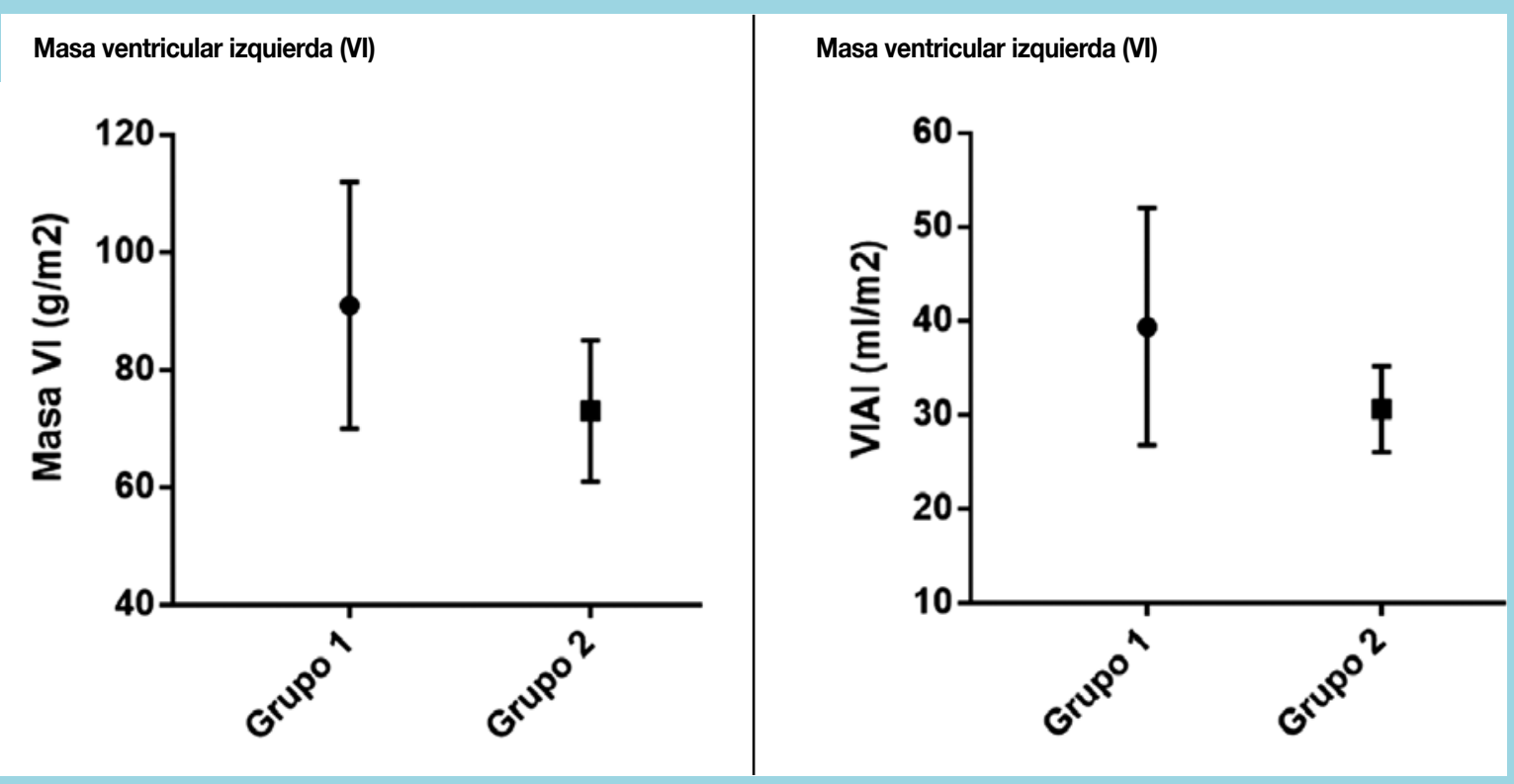

G1: Grupo 1 Alta carga de entrenamiento. G2: Grupo baja carga de entrenamiento VIAl: volumen indexado auricular izquierdo

\section{Conclusiones:}

Posterior a un esfuerzo intenso se produce un aumento de los niveles de IL-6 y MDA. Los atletas mejor entrenados presentaron una menor respuesta de estrés oxidativo frente a una maratón y, además, manifestaron una tendencia no significativa a tener menores niveles basales de inflamación (IL-6). Finalmente, respecto a las variables ecocardiográficas estudiadas, ambos grupos presentaron similar función sistólica bi-ventricular y deformación del ventrículo izquierdo. Sin embargo, el grupo mejor entrenado presentó un mayor remodelado del corazón izquierdo, evidenciado por una mayor masa del ventrículo izquierdo y mayor volumen de la aurícula izquierda.

\section{Limitaciones:}

Nuestro trabajo tiene limitaciones relacionadas al redu- cido tamaño de la muestra, la ausencia de seguimiento posterior a los atletas (en seguimiento actualmente), la exclusiva participación de atletas de sexo masculino y que es incierto si los resultados son extrapolables a otra disciplina deportiva. En cuanto a la evaluación del estrés oxidativo, no está objetivada actualmente la actividad antioxidante total de cada atleta, que se asume mayor en el grupo mejor entrenado. Eso puede ser uno de los mecanismos que frenaría el alza del MDA, aspectos que son actualmente materia de investigación.

Financiamiento: Este trabajo fue financiado por Fondecyt 1170963 (LG) y Fondap ACCDiS 15130011 (LG, MC, MPO, SL, PC).

Agradecimientos: A Empresas COPEC S.A., y a su presidente, Sr. Roberto Angelini. 


\section{Referencias}

1. MORRIS JN, CRAWFORD MD. Coronary heart disease and physical activity of work; evidence of a national necropsy survey. Br Med J. 1958;2:1485-1496.

2. GEBEL K, DING D, CHEY T, STAMATAKIS E, BROWN WJ, BAUMAN AE. Effect of Moderate to Vigorous Physical Activity on All-Cause Mortality in Middle-aged and Older Australians. JAMA Intern Med. 2015;175:970.

3. AREM H, MOORE SC, PATEL A, HARTGE P, BERRINGTON DE GONZALEZ A, VISVANATHAN K, et al. Leisure Time Physical Activity and Mortality. JAMA Intern Med. 2015;175:959.

4. PELLICCIA A, KINOSHITA N, PISICCHIO C, QUATTRINI F, DIPAOLO FM, CIARDO R, et al. Long-Term Clinical Consequences of Intense, Uninterrupted Endurance Training in Olympic Athletes. J Am Coll Cardiol. 2010;55:1619-1625.

5. SHARMA S, MERGHANI A, MONT L. Exercise and the heart: The good, the bad, and the ugly. Eur Heart J. 2015;36:14451453.

6. COHN JN, FERRARI R, SHARPE N. Cardiac remodelingconcepts and clinical implications: a consensus paper from an international forum on cardiac remodeling. J Am Coll Cardiol. 2000;35:569-582.

7. D'ASCENZI F, PELLICCIA A, CORRADO D, CAMELI M, CURCI V, ALVINO F, et al. Right ventricular remodelling induced by exercise training in competitive athletes. Eur Hear J - Cardiovasc Imaging. 2016;17(3):301-307.

8. CASELLI S, PELLICCIA A. Interpretation of left ventricular geometry in athletes. Int J Cardiol. 2018;267:143-4.

9. D'ASCENZI F, PELLICCIA A, NATALI BM, BONIFAZI M, MONDILLO S. Exercise-induced left-ventricular hypertrabeculation in athlete's heart. Int J Cardiol. 2015;181:320-2.

10. TRIVAX JE, MCCUllOUGH PA. Phidippides Cardiomyopathy: A Review and Case Illustration. Clin Cardiol. 2012;35:69-73.

11. BENITO B, GAY-JORDI G, SERRANO-MOLLAR A, GUASCH E, SHI Y, TARDIF J-C, et al. Cardiac Arrhythmogenic Remodeling in a Rat Model of Long-Term Intensive Exer- cise Training. Circulation. 2011;123:13-22.

12. ABDULLA J, NIELSEN JR. Is the risk of atrial fibrillation higher in athletes than in the general population? A systematic review and meta-analysis. Europace. 2009;11:1156-9.

13. MOHLENKAMP S, LEHMANN N, BREUCKMANN $\mathrm{F}$, BROCKER-PREUSS M, NASSENSTEIN K, HALLE M, et al. Running: the risk of coronary events : Prevalence and prognostic relevance of coronary atherosclerosis in marathon runners. Eur Heart J. 2008;29:1903-10.

14. SHAVE R, BAGGISH A, GEORGE K, WOOD M, SCHARHAG J, WHYTE G, et al. Exercise-Induced Cardiac Troponin Elevation: Evidence, Mechanisms, and Implications. J Am Coll Cardiol. 2010;56:169-76.

15. SCHERR J, BRAUN S, SCHUSTER T, HARTMANN C, MOEHLENKAMP S, WOLFARTH B, et al. 72-h Kinetics of High-Sensitive Troponin $\mathrm{T}$ and Inflammatory Markers after Marathon. Med Sci Sport Exerc. 2011;43:1819-27.

16. CHILD RB, WILKINSON DM, FALLOWFIELD JL, DONNELLY AE. Elevated serum antioxidant capacity and plasma malondialdehyde concentration in response to a simulated half-marathon run. Med Sci Sports Exerc. 1998;30:1603-7.

17. FISCHER CP. Interleukin-6 in acute exercise and training: what is the biological relevance? Exerc Immunol Rev. 2006;12:6-33.

18. SHEPHARD RJ. Cytokine responses to physical activity, with particular reference to IL-6: sources, actions, and clinical implications. Crit Rev Immunol. 2002;22:165-82.

19. PEDERSEN BK, STEENSBERG A, SCHJERLING P. Exercise and interleukin-6. Curr Opin Hematol. 2001;8:137-41.

20. GOKHALE R, CHANDRASHEKARA S, VASANTHAKUMAR KC. Cytokine response to strenuous exercise in athletes and non-athletes-an adaptive response. Cytokine. 2007;40:123-7.

21. AZIZBEIGI K, STANNARD SR, ATASHAK S, MOSALMAN HAGHIGHI M. Antioxidant enzymes and oxidative stress adaptation to exercise training: Comparison of endurance, resistance, and concurrent training in untrained males. J Exerc Sci Fit. 2014;12:1-6. 
22. RADÁK Z, KANEKO T, TAHARA S, NAKAMOTO H, OHNO H, SASVÁRI M, et al. The effect of exercise training on oxidative damage of lipids, proteins, and DNA in rat skeletal muscle: evidence for beneficial outcomes. Free Radic Biol Med. 1999;27:69-74.

23. DILLARD CJ, LITOV RE, SAVIN WM, DUMELIN EE, TAPPEL AL. Effects of exercise, vitamin E, and ozone on pulmonary function and lipid peroxidation. J Appl Physiol. 1978;45:927-32.

24. POWERS SK, RADAK Z, JI LL. Exercise-induced oxidative stress: past, present and future. J Physiol. 2016;594:5081-92.

25. GOMEZ-CABRERA M-C, MARTÍNEZ A, SANTANGELO
G, PALLARDÓ F V, Sastre J, Viña J. Oxidative stress in marathon runners: interest of antioxidant supplementation. Br J Nutr. 2006;96:S31-3.

26. SCOTT JPR, SALE C, GREEVES JP, CASEY A, DUTTON J, FRASER WD. Cytokine response to acute running in recreationally-active and endurance-trained men. Eur J Appl Physiol. 2013;113:1871-82.

27. SCHILD M, EICHNER G, BEITER T, ZÜGEL $M$, KRUMHOLZ-WAGNER I, HUDEMANN J, et al. Effects of Acute Endurance Exercise on Plasma Protein Profiles of Endurance-Trained and Untrained Individuals over Time. Mediators Inflamm. 2016:1-11. 